\title{
SEROTONIN SYNDROME MASKED BY CONCOMITANT INGESTION OF ZOLOFT WITH BENZODIAZEPINE IN SUICIDE ATTEMPT IN A YOUNG HEALTHY MALE
}

\author{
Kelly Schrapp and Walid Omer \\ Department of Internal Medicine, Morehouse School of Medicine, Atlanta, GA 30310-1495, Georgia
}

Received 2014-04-09; Revised 2014-04-14; Accepted 2014-04-30

\begin{abstract}
This case report discusses the history and management of a schizophrenic patient, a 18-year-old man, who had taken a massive overdose of clonazepam, abilify, depakote, zoloft and seroquel altogether. After discontinuation of these drugs and administration of cyproheptadine with IV fluids, he became hemodynamically stable. This case suggests that serotonin syndrome should be recognized promptly and complications need to be treated appropriately. Serotonin syndrome should be diagnosed surely to ensure the prompt initiation of the treatment including discontinuation of the precipitating drugs because the disease occasionally progresses rapidly to fatal condition. In addition, both the onset and resolution of symptoms are faster in serotonin syndrome.
\end{abstract}

Keywords: Serotonin Syndrome, Adverse Drug Reaction, Antidepressants, Drug Interaction

\section{INTRODUCTION}

The serotonin syndrome is the clinical demonstration of serotonin toxicity in patients taking one or more serotonergic agents. The serotonin syndrome has a potentially severe effect caused by serious serotonergic activity in the central nervous system. It is well-known by features of (1) mental status changes such as confusion, agitation, lethargy and coma, (2) autonomic instability such as hyperthermia, tachycardia, diaphoresis, nausea, vomiting, diarrhea and dilated pupils) and (3) neuromuscular hyperactivity such as myoclonus, hyperreflexia, rigidity and trismus. This disease is a manifestation of an overload of serotonin and include a range of clinical features ranging from minor symptoms to death.

The syndrome is instigated by the driving force of a subset of serotonin receptors in the brainstem and spinal cord. Almost all cases reported were exposed to a number of serotonergic drugs, for example Selective
Serotonin Reuptake Inhibitors (SSRI), Tricyclic Antidepressants (TCA) and Monoamine Oxidase Inhibitors (MAOI).

Diagnosis is recognized on clinical presentation and exposure to serotonergic medications, though other roots of mental changes characteristically need to be excluded. Management consists of termination of the offending agent and supportive care, although a possible medication with serotonin antagonists, such as cyproheptadine and benzodiazepine can aid in validating the diagnosis and improve symptoms. Medication overdose can trigger serotonin syndrome through a range of mechanisms: (1) Blockade of serotonin reuptake; (2) inhibition of serotonin metabolism; (3) increased production of serotonin and (4) increased serotonin release.

Combinations of atypical antipsychotics, such as risperidone (Mathew et al., 1996) or olanzapine (Bernard et al., 2003), added to a sselective serotonin reuptake inhibitors have instigated serotonin syndrome. Atlanta, GA 30310-1495, Georgia 
Both analgesics, meperidine and tramadol, are also serotonin receptor agonists. A specific amount of meperidine can cause serotonin syndrome in a patient getting fluoxetine (Pan and Shen, 2003), along with other many cases of tramadol instigating this disorder after the addition of different serotonin reuptake inhibitors (Bodnar et al., 1998). The authors are the first to report a shortened and less severe course of serotonin syndrome due to the patient overdosing with a benzodiazepine.

\section{CASE REPORT}

An 18-year-old male was brought to the emergency department due to confusion and sudden, involuntary movements. He was found with an empty bottle of seroquel next to him. The patient was diagnosed previously with schizophrenia and was taking Zoloft, abilify, depakote and clonazepam. The patient's psychiatrist had recently decreased his medication to seroquel $100 \mathrm{mg}$ daily alone. The patient was last seen normal the previous night at $9 \mathrm{pm}$ and was found confused with involuntary movements in the morning by his sister and was brought to our hospital because of confusion. He had taken no other medications or dietary supplements.

He denied using illicit drugs and alcohol. The patient's psychotropic medications were stopped and he was admitted to the intermediate care unit for further observation, stabilization and diagnostic workup.

On examination the patient was confused and had hyperhidrosis, hyperreflexia, myoclonus and mydriasis. There were no focal neurological findings. His blood pressure was $163 / 84 \mathrm{mmHg}$, heart rate 172 beats/min, respiratory rate 13 breaths/min and temperature $37.7 \mathrm{C}$. Blood gas analysis, electrolytes, liver and kidney function tests and total creatine kinase level were normal. EKG showed sinus tachycardia on admission (Fig. 1). Chest Xray and a brain CT scan showed no abnormalities. Qualitative plasma tests for carbamates, salicylates, paracetamol, barbiturates and triycyclic antidepressants were negative. The Qualitative plasma test was positive for Benzodiazepines. No plasma concentration of Zoloft, seroquel, abilify and depakote were available.

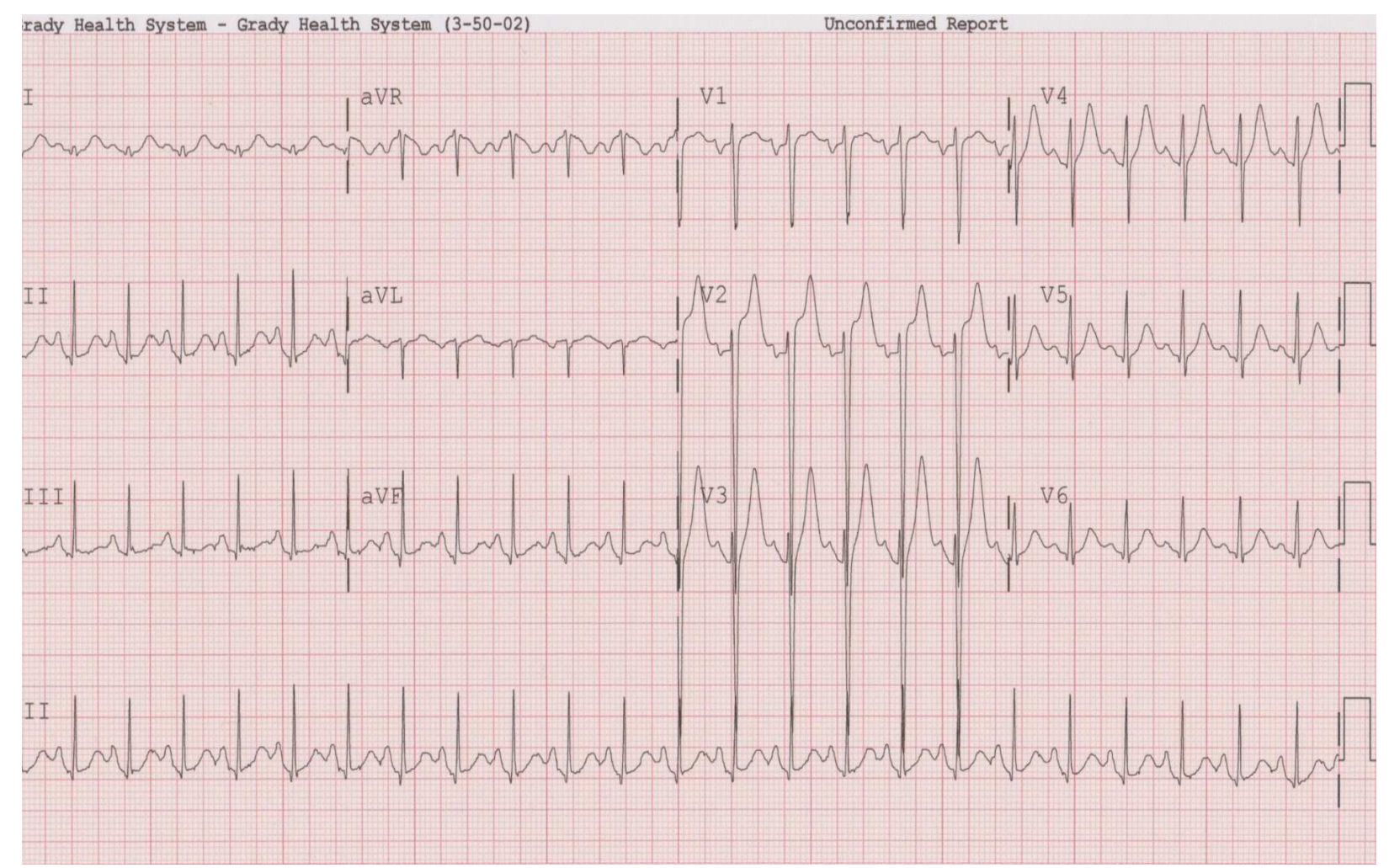

(a) 


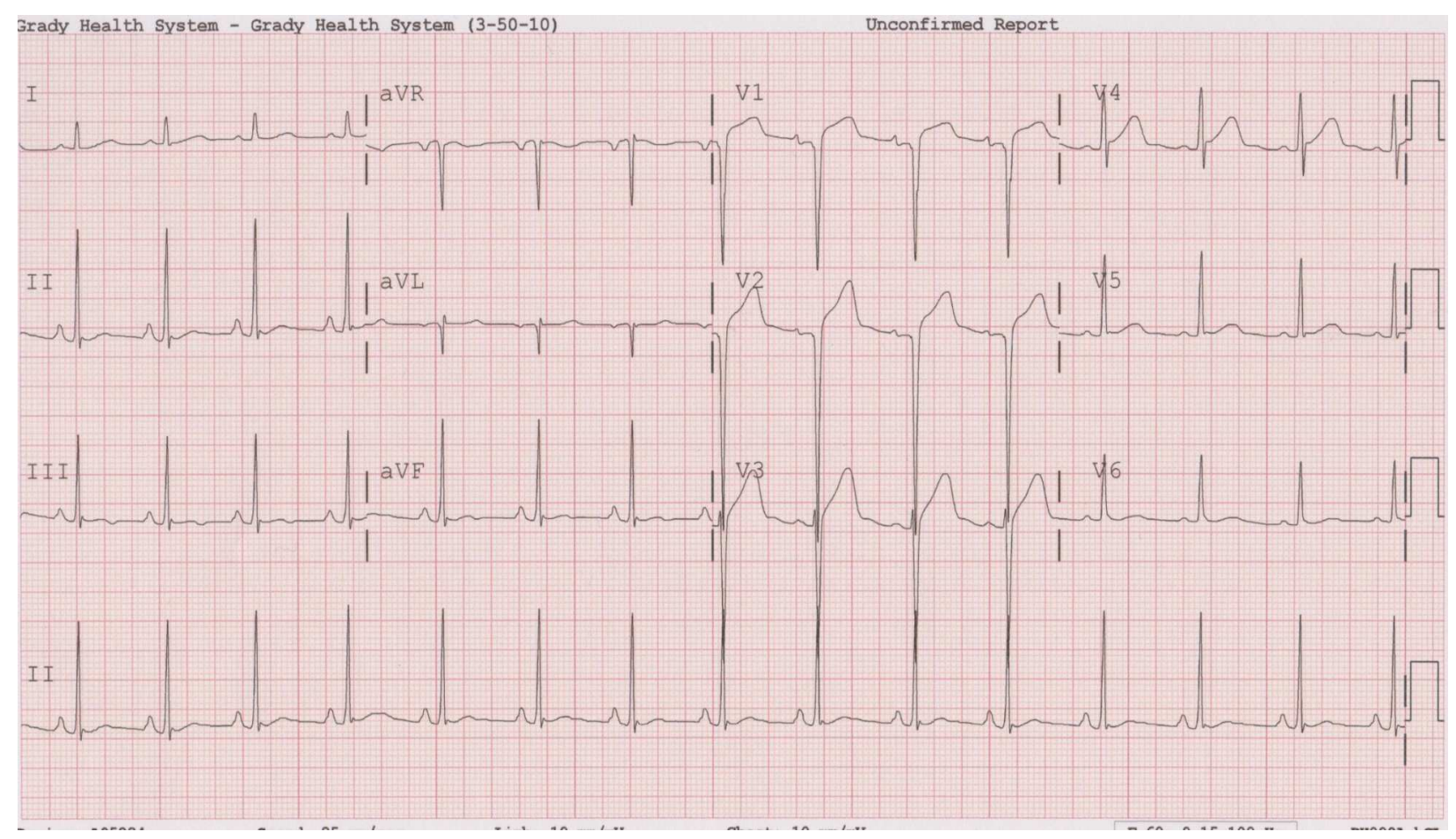

(b)

Fig. 1. Electrocardiography (EKG) on and after admission (A) on admission shows sinus tachycardia; (B) on discharge shows normal sinus rhythm

During the subsequent evening, the patient was given Normal Saline $200 \mathrm{~mL} \mathrm{~h}^{-1}$, cyproheptadine 4 $\mathrm{mg}$ TID were required to prevent severe increases in both blood pressure and heart rate, as well as to control subjective complaints of restlessness/akathesia. He wasn't given benzodiazepines as part of the treatment due to the fact that he overdosed on benzodiazepine. By the next day, it was possible to wean the patient from cyproheptadine and intravenous fluids and no increases in heart rate or pulse were observed. The patient's tremor, diaphoresis, nystagmus and hyperreflexia had markedly attenuated and gradually resolved after several days. The patient was evaluated by psychiatry and was restarted on his seroquel alone as he was having suicidal ideation.

Patient became more alert and admitted to ingestion 30 tablets that included a mixture of clonazepam, abilify, depakote, zoloft and Seroquel 30-40 pills altogether. Mood stabilizer therapy was reinitiated and the patient was discharged to an outpatient psychiatric provider.

\section{DISCUSSION}

Our patient met the diagnostic criteria for serotonin syndrome according to Sternbach's interpretation. He showed signs of six of the symptoms normally linked with serotonin syndrome: (1) Agitation, (2) diaphoresis, (3) clonus, (4) hyperreflexia, (5) hypertonicity and (6) tremor following the increased ingestion of a serotonergic agent. Alternative diagnoses were ruled out either by history or by laboratory assessment.

Differential diagnosis of serotonin syndrome consists of Neuroleptic Malignant Syndrome (NMS), dystonic reaction, hyperthyroidism, tetanus, malignant hyperthermia and other disorders that produce muscle rigidity and includes toxicities of certain drugs such as cocaine, monoamine oxidase inhibitors, amphetamines, lithium and other drugs (Dunkley et al., 2003). Patients with serotonin syndrome are commonly misdiagnosed with NMS (Birmes et al., 2003). This is in particular accurate especially with patients receiving both neuroleptic and serotonergic agents. NMS is repeatedly confounded with serotonin syndrome for the reason that 
mental status changes, autonomic dysfunction, along with neuromuscular abnormalities illustrate both syndromes. The only characteristic that distinguished NMS from serotonin syndrome is the lead-pipe stiffness related with the previous syndrome distinguished with the clonus and hyperreflexia observed with serotonin syndrome. Collectively, the commencement and resolution of symptoms are more rapidly in serotonin syndrome (Dunkley et al., 2003). In our patient, the rigorous autonomic alteration and muscle stiffness made the diagnosis of NMS unlikely (Birmes et al., 2003). Additionally, there was no confirmation of the other differential diagnoses explained above.

Furthermore, Zoloft is a selective serotonin reuptake inhibitor (SSRI) antidepressant to facilitate production of a net increase in 5-HT mediated transmission. Also, Clonazepam is a benzodiazepine which boosts the effect of the neurotransmitter Gamma-Amino Butyric Acid (GABA) that operates on the GABA receptor and has a sedative, hypnotic effect and abilify and seroquel are atypical antipsychotic agents which are partial dopamine agonists. Depakote is an anticonvulsant and used as a mood stabilizer. Co-administration of Zoloft, Abilify, Seroquel, Depakote and clonazepam has not been documented to result in serotonin syndrome. The patient received heavy doses of all of these medications the night before and the next morning was found to have AMS, agitation, diaphoresis and tremors.

Management of serotonin syndrome comprises discontinuation of the offending serotonergic agent, supportive therapy and, most likely, the use of serotonin antagonists (Thanacoody, 2012). Our patient improved gradually within 2 days after Zoloft, Abilify, seroquel, depakote and clonazepam was discontinued. His condition improved completely after several more days.

Even though the above medications are comparatively safe to use, serotonin syndrome needs to be considered when prescribing these drugs alone or in a mixture with other drugs. With the increased utiliziation of SSRIs in the treatment of psychiatric disorders, the significance of distinguishing this disorder cannot be exaggerated. In our patient, he overdosed on SSRI, atypical antipsychotics and an anticonvulsant in elevated doses. These high doses of drugs are widespread for causing serotonin syndrome. We suggest this drug overdose in combination with a benzodiazipine may have lessened the degree of serotonin syndrome in our patient developed as it is the part of the treatment. However, also worsened his CNS state and respiratory state on admission.

Finally, serotonin toxicity results from an overload of serotonin in the CNS, which can be due to inhibition of the metabolism of serotonin, prevention of the reuptake of serotonin into nerve terminals, increased serotonin precursors or increased serotonin release (Isbister et al., 2007).

\section{CONCLUSION}

Our patient likely had a lessened degree of serotonin syndrome due to the fact that he also overdosed on a benzodiazepine which is used for treatment. Benzodiazepines are central to the control of agitation, regardless of syndrome severity. Management of serotonin syndrome begins with discontinuation of all serotonergic agents. Supportive care is the treatment of choice for most cases, but specific therapies depend on the severity of the syndrome. Mild cases are usually managed by removal of precipitating agents, as well as supportive care including intravenous fluids for hydration and to facilitate diuresis and treatment with benzodiazepines to prevent agitation.

\section{REFERENCES}

Bernard, L., R. Stern, D. Lew and P. Hoffmeyer, 2003. Serotonin syndrome after concomitant treatment with linezolid and citalopram. Clin. Infect. Dis., 36: 1197-1197. DOI: 10.1086/374558

Birmes, P., D. Coppin, L. Schmitt and D. Lauque, 2003. Serotonin syndrome: A brief review. CMAJ, 168: 1439-42.

Bodnar, A.G., M. Ouellette, M. Frolkis, S.E. Holt and C.P. Chiu et al., 1998. Extension of life-span by introduction of telomerase into normal human cells. Science, 279: 349-352 DOI: 10.1126/science. 279.5349 .349

Dunkley, E.J.C., G.K. Isbister, D. Sibbritt, A.H. Dawson and I.M. Whyte, 2003. The hunter serotonin toxicity criteria: Simple and accurate diagnostic decision rules for serotonin toxicity. QJM, 96: 635-42. DOI: 10.1093/qjmed/hcg109

Isbister, G.K., N.A. Buckley and I.M. Whyte, 2007. Serotonin toxicity: A practical approach to diagnosis and treatment. Med. J. Aust., 187: 361-5.

Mathew, N.T., G.E. Tietjen and C. Lucker, 1996. Serotonin syndrome complicating migraine pharmacotherapy. Cephalalgia, 16: 323-327. PMID: 8869767

Pan, J.J. and W.W. Shen, 2003. Serotonin syndrome induced by low-dose venlafaxine. Ann. Pharmacother., 37: 209-211. DOI: 10.1345/aph.1C021

Thanacoody, R., 2012. Serotonin syndrome. Medicine, 40: 63-64. DOI: 10.1016/j.mpmed.2011.11.005 